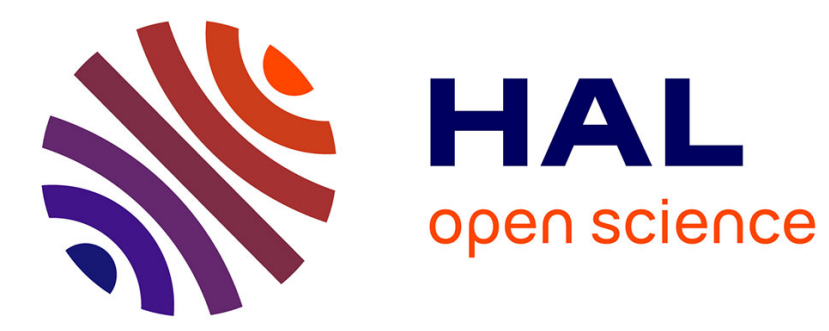

\title{
Control and automation for miniaturized microwave GSG nanoprobing
}

Alaa Taleb, Denis Pomorski, Christophe Boyaval, S. Arscott, Gilles Dambrine, Kamel Haddadi

\section{- To cite this version:}

Alaa Taleb, Denis Pomorski, Christophe Boyaval, S. Arscott, Gilles Dambrine, et al.. Control and automation for miniaturized microwave GSG nanoprobing. Machine Vision and Navigation, Springer International Publishing, pp.751-768, 2020, 10.1007/978-3-030-22587-2_24 . hal-02345340

\section{HAL Id: hal-02345340 \\ https://hal.science/hal-02345340}

Submitted on 31 Aug 2021

HAL is a multi-disciplinary open access archive for the deposit and dissemination of scientific research documents, whether they are published or not. The documents may come from teaching and research institutions in France or abroad, or from public or private research centers.
L'archive ouverte pluridisciplinaire HAL, est destinée au dépôt et à la diffusion de documents scientifiques de niveau recherche, publiés ou non, émanant des établissements d'enseignement et de recherche français ou étrangers, des laboratoires publics ou privés. 


\title{
Control and Automation for Miniaturized Microwave GSG Nanoprobing
}

\author{
A. Taleb \\ Univ. Lille, CNRS, Centrale Lille, UMR 9189-CRIStAL — Centre de Recherche en \\ Informatique, Signal et Automatique de Lille, Lille, France \\ D. Pomorski ( $\triangle)$ \\ Univ. Lille, CNRS, Centrale Lille, UMR 9189-CRIStAL_Centre de Recherche en \\ Informatique, Signal et Automatique de Lille, Lille, France \\ Univ. Lille, IUT A-Département GEII, Lille, France \\ e-mail: denis.pomorski@univ-lille.fr \\ C. Boyaval · S. Arscott · G. Dambrine \\ Univ. Lille, CNRS, Centrale Lille, ISEN, Univ. Valenciennes, UMR 8520_IEMN, Lille, France \\ e-mail: christophe.boyaval@univ-lille.fr; steve.arscott@univ-lille.fr; \\ gilles.dambrine@univ-lille.fr \\ K. Haddadi \\ Univ. Lille, CNRS, Centrale Lille, ISEN, Univ. Valenciennes, UMR 8520_IEMN, Lille, France \\ Univ. Lille, IUT A-Département GEII, Lille, France \\ e-mail: kamel.haddadi@univ-lille.fr
}




\subsection{Introduction}

\subsubsection{Context}

To drive the progress of the miniaturization of electronic circuits, new metrological issues related to the dimensional and electrical characterization of nanoelectronic devices must be addressed [1]. In addition, the electrical characterization of highimpedance $1 \mathrm{D}$ or $2 \mathrm{D}$ based nanodevices in the microwave regime is still challenging [2]. A typical high-frequency (HF) device characterization is built up with a vector network analyzer, a probe station equipped with a pair of microwave GSG probes aligned manually through a microscope or a camera system onto calibration substrates and test devices $[3,4]$. Conventional HF test structures require probing pads around $50 \times 50 \mu^{2}$ to accommodate the probe tip geometry (center-tocenter pitch of $100 \mu \mathrm{m}$, contact area of $20 \times 20 \mu \mathrm{m}^{2}$ ). The extrinsic parasitic capacitance associated with the pad in the range of $50 \mathrm{fF}$ is therefore not compatible to address the metrology of nanodevices. Furthermore, actual visualization and displacement/positioning techniques are not accurate enough to ensure a repeatable contact between the probe tips and the pads at the micro- and nanoscale.

Intensive research has been described in the literature to address RF metrology at the nanoscale. In 2005, the first measurements of the high-frequency conductance of a metallic single-walled nanotube (SWNT) with resistance below $200 \mathrm{k} \Omega$ inserted in a coplanar waveguide (CPW) transmission line were performed up to $10 \mathrm{GHz}$ [5]. In 2008, an on-wafer technique and calibration method are developed for broadband electrical characterization of $\mathrm{GaN}$ nanowires up to $40 \mathrm{GHz}$ [6]. In 2010, to improve the vector network analyzer (VNA) sensitivity, an individual SWNT is inserted in a specific high impedance Wheatstone bridge helps to reduce the impedance mismatch between the VNA and the high-impedance nanodevice [7]. Other indirect measurements including nanotube transistors acting as resonators [8] or microwave detectors [9] have been proposed to demonstrate their $\mathrm{GHz}$ operation. Despite these pioneering works, in the era of shrinking GSG probing structures, a gap between commercially available probes and those required to characterize nanodevices still remains.

The objective of this work is to develop a new generation of on-wafer probing instrumentation dedicated to $\mathrm{HF}$ quantitative characterization of microand nanodevices. At such scale, visualization, accuracy of alignment, positioning, and repeatability require suited techniques. In the solution proposed, the probes are mounted on nanopositioners, and the visualization is ensured by a scanning electron microscopy (SEM) rather than optics. This method suffers from measurement repeatability and accuracy issues. Consequently, we have developed a unique instrument that is a compromise between conventional on-wafer probe station and microscopy tools. We have fabricated microelectromechanical system (MEMS) technology-based miniaturized microwave ground-signal-ground (GSG) probes 
[10]. In contrast to conventional macroscopic on-wafer probing structures, micrometric CPW test structures have been designed and fabricated to accommodate the miniaturized probes and to ensure quasi-transverse electromagnetic (quasi-TEM) mode propagation to the nanoscale devices embedded in the test structures [11]. The probes are mounted on nanopositioners, and imaging is ensured by an SEM. A detailed study on the development of the nanorobotic on-wafer probe station is given in [12].

\subsubsection{Short Description of the SEM}

The SEM [13] (Fig. 24.1) consists of observing the topography of a surface (a substrate). Its operation relies essentially on the detection of the secondary electrons (1) emerging from the observed surface (2) under the impact of a primary electron beam (3) which scans it. The images obtained from the substrate (Fig. 24.2) have a separating power often less than $5 \mathrm{~nm}$ and a great depth of field.

Without going into details of the internal functioning of the SEM, the general idea of this work consists of positioning a probe consisting of three aligned points on an element (pattern) of the substrate (Fig. 24.2). It is therefore a system with four degrees of freedom requiring the control of the displacements of three nanopositioners SmarAct ${ }^{\mathrm{TM}}$ (Fig. 24.3) [14] in $X, Y$, and $Z$ and the control of one nanopositioner around an axis of rotation $\theta$.



Fig. 24.1 Schematic layout of the SEM 




Fig. 24.2 SEM image of the substrate

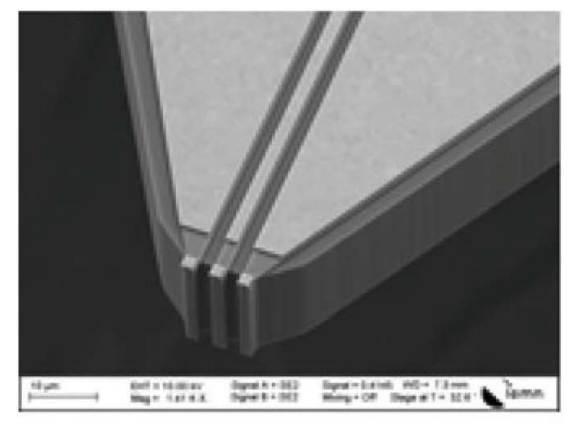

(a)

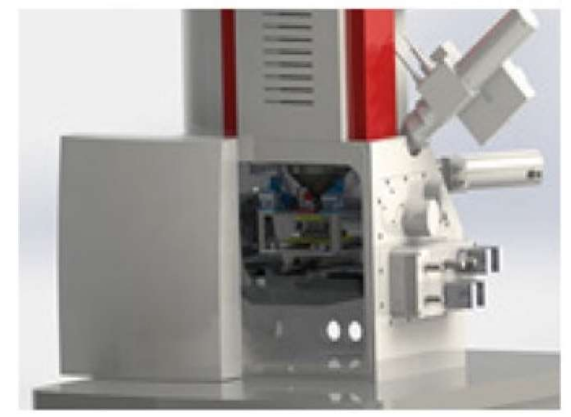

(c)

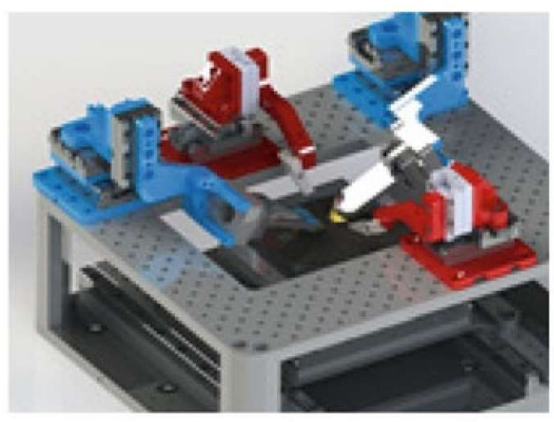

(b)

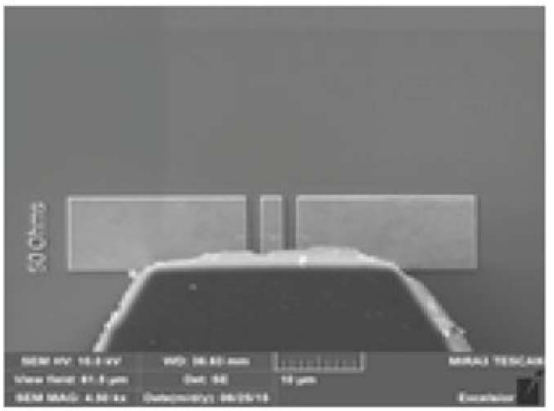

(d)

Fig. 24.3 (a) SEM image of the probe. (b) Probe nanopositioning platform. (c) Scanning electron microscope Tescan Mira XMU. (d) SEM image of the measuring probe which is in contact with a test structure 


\subsubsection{Specifications}

Our specifications are as follows:

First, we propose to model and control any linear nanopositioners. In this study, we focus on three linear nanopositioners $X, Y$, and $Z$ (Sect. 24.2).

Both linear nanopositioners $X$ and $Y$ can be controlled by taking into account a minimum response time. The linear nanopositioner $Z$ must be controlled without overshooting the set point (in order to avoid any crashing of the probe tips on the DUT).

Section 24.3 provides an approach for controlling the nanopositioner in $\theta$ in order to align the probe on the image.

Finally, a simple approach for detecting points of interest (Harris method) allows determining the set point value of each nanopositioner in $X, Y$, or $Z$ (Sect. 24.4).

The overall process allows us to position the probe accurately at any point of the substrate.

\subsection{Modeling and Control of a Linear Nanopositioner Using LabVIEW ${ }^{\text {TM }}$}

\subsubsection{Central Idea of This Study}

The main idea of this study is to fully master the nanomanipulator control chain from two elementary blocks using LabVIEW ${ }^{\mathrm{TM}}$ (Fig. 24.4) [15, 16]:

- A set point or control block

- A block for acquiring the actual position of the nanopositioner

These two elements allow a real-time interaction with the nanopositioner (Fig. 24.5)

So we can use a conventional control loop to control the nanomanipulator (Fig. 24.6). A HIL (Hardware-In-the-Loop) test is thus carried out.
(a)


(b)



Fig. 24.4 Blocks used with LabVIEW ${ }^{\mathrm{TM}}$ : (a) Set point block. (b) Position acquisition block

Fig. 24.5 Control of a nanopositioner with LabVIEWTM

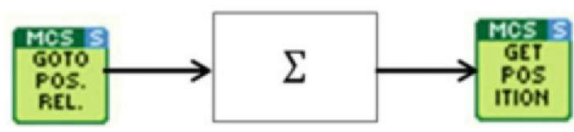




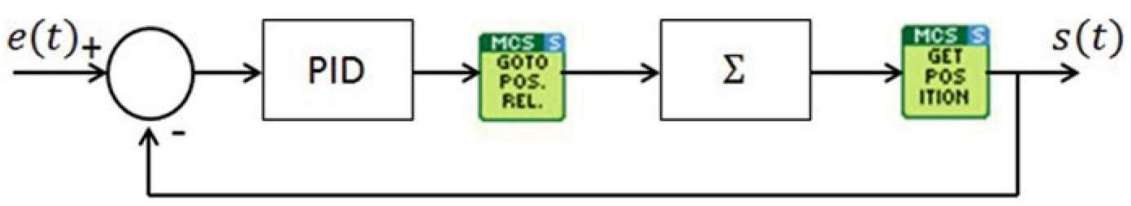

Fig. 24.6 Control loop for the nanopositioner, where $e(t)$ represents the required position of the nanopositioner-it is the set point; $s(t)$ is its measured/actual position; the PID controller continuously applies a correction based on proportional, integral, and derivative terms from the error value $\varepsilon(t)$ which is the difference between the required position (the set point $e(t)$ ) and the measured/actual position $s(t)$

$$
\stackrel{E(s)}{\longrightarrow} T(s) \longrightarrow S(s)
$$

Fig. 24.7 Open-loop transfer function of the nanopositioner, where $E(s)$ is the Laplace transform of the required (desired) position $e(t) ; S(s)$ is the Laplace transform of the actual position $s(t) ; T(s)$ is the transfer function of the nanopositioner. $T(s)$ represents the Laplace transform of the impulse response of the nanopositioner

In order to obtain an accurate control of the nanomanipulator, we must identify its transfer function. We considered that the system is linear. This assumption is not contradicted by the experiments.

We propose to identify its linear transfer function using a (or some) basic identification method(s).

\subsubsection{Modeling}

After testing two open-loop identification methods, we propose a more accurate closed-loop identification technique.

\subsubsection{Identification of the Open-Loop Transfer Function of the Nanopositioner}

First, let us try to identify the open-loop transfer function of the nanopositioner (Fig. 24.7).

For instance, the set point value is fixed at $1000 \mathrm{~nm}$. Figure 24.8 shows the required position (in red) and the actual position (in blue) of the nanopositioner with respect to time. For a greater clarity of the curves, the $\mathrm{X}$-axis is graduated in tenths of a second ( 1 unit $\left.=\frac{1}{10} \mathrm{~s}\right)$, which represents the sampling period of the measured position.

The nanopositioner reacts globally as a pure integrator. This property allows us to assume that the closed-loop permanent error (difference between the output and 


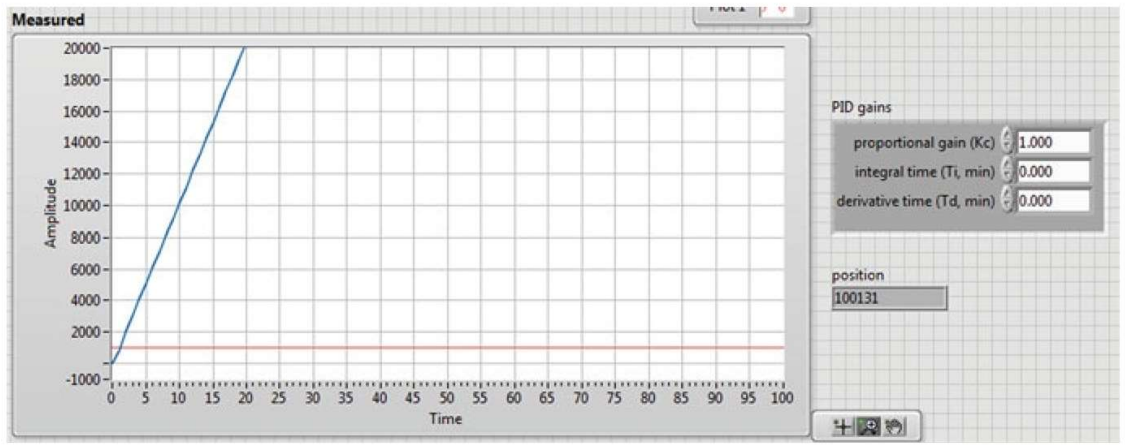

Fig. 24.8 Open-loop response with a set point of $1000 \mathrm{~nm}$ of the nanopositioner

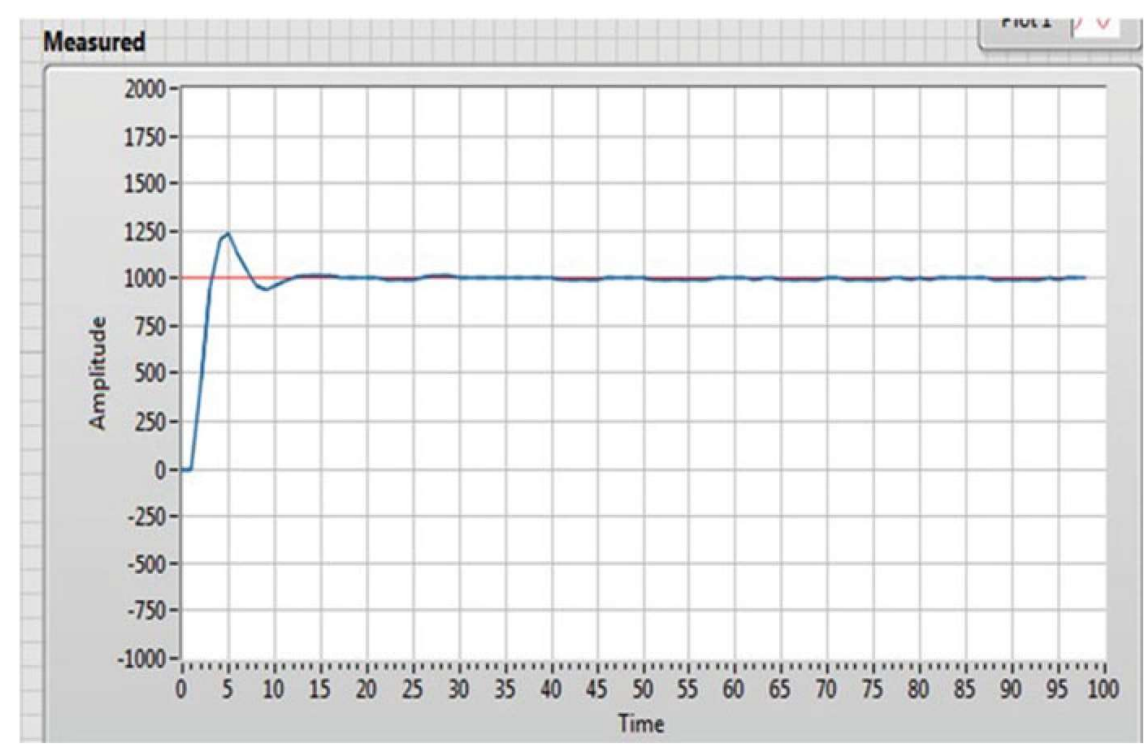

Fig. 24.9 Closed-loop response of the nanopositioner for a set point of $1000 \mathrm{~nm}$

the set point for a constant set point value when $t \rightarrow \infty$ ) is zero. This is confirmed by the actual closed-loop response (Fig. 24.9) without PID controller (or with a proportional controller with a gain factor equal to 1).

A proportional correction seems sufficient (Fig. 24.10).

The identification of the nanopositioner transfer function can be performed only by the hypothesis of a mathematical expression. 


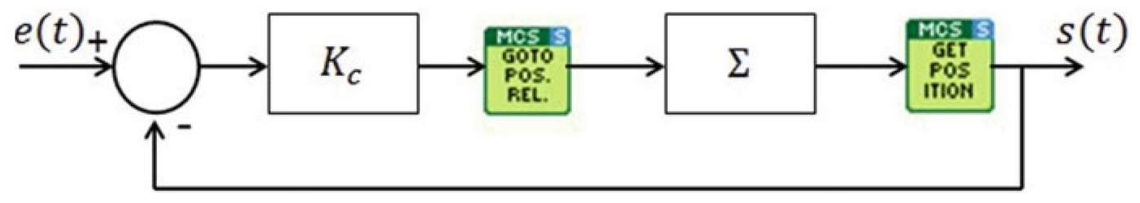

Fig. 24.10 A proportional correction of the nanopositioner

\section{First hypothesis}

If we consider that the nanopositioner behaves as a pure integrator, its transfer function is as follows:

$$
T(s)=\frac{K}{s}
$$

From the identification, we obtain $K=1.05$.

However, the closed-loop nanopositioner shows that for some values of $K_{\mathrm{c}}$, there is an overshoot of the set point value (e.g., Fig. 24.9).

Consequently, the first hypothesis must be rejected, in favor of a second-order model at least.

\section{Second hypothesis}

The transfer function is as follows:

$$
T(s)=\frac{K}{s(1+\tau s)}
$$

The expression " $(1+\tau s)$ " will have a preponderant role only when $t \rightarrow 0$ and it zan be considered as a time delay (dead time $\tau$ ), using the Taylor series expansion: $e^{-\tau s} \sim \frac{1}{1+\tau s}$.

From the previous identification, $\tau=1$ unit $=100 \mathrm{~ms}$. The transfer function thus becomes

$$
T(s)=\frac{1.05}{s(1+s)}
$$

However, the closed-loop responses of the model (Fig. 24.11) and the real system (Fig. 24.9) are too distinct to consider the model to be correct.

We can conclude that the method used to identify the open-loop transfer function is not accurate, given the sampling period of $100 \mathrm{~ms}$. We have therefore chosen to identify the parameters $K$ and $\tau$ differently using an identification of the closed-loop transfer function. 


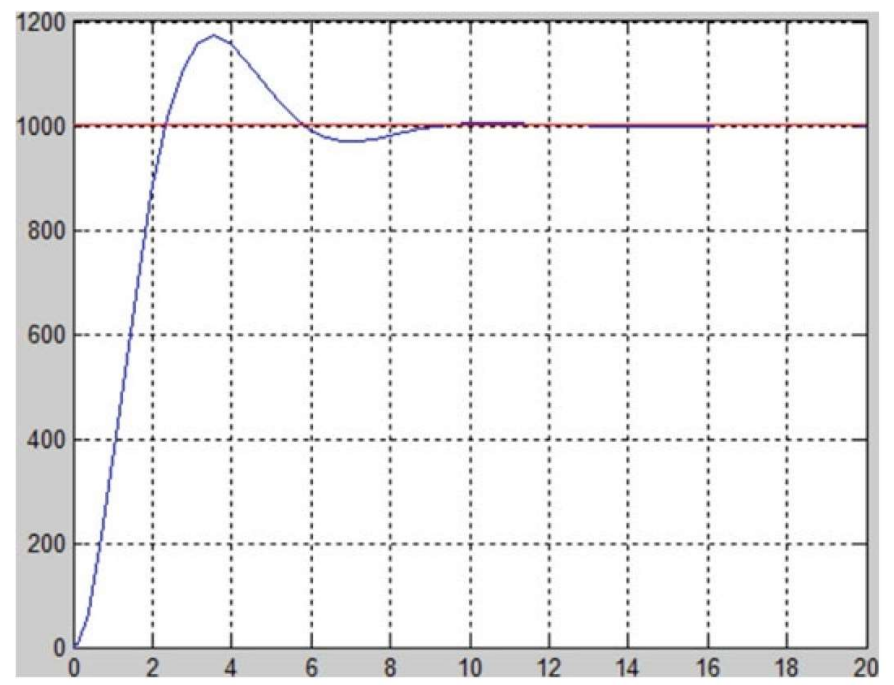

Fig. 24.11 Closed-loop response of the model (Eq. 24.3) for a set point of $1000 \mathrm{~nm}$

\subsubsection{Nanopositioning in Closed Loop}

Taking into account the second hypothesis for the open-loop transfer function $T(s)=$ $\frac{K}{s(1+\tau s)}$ and a proportional controller with a gain factor equal to $K_{\mathrm{c}}$, the closed-loop transfer function of the nanopositioner is as follows:

$$
H(s)=\frac{S(s)}{E(s)}=\frac{1}{1+\frac{1}{K K_{\mathrm{c}}} s+\frac{\tau}{K K_{\mathrm{c}}} s^{2}}
$$

We can rely on the well-known equations of the automatic control of a linear system:

- The canonical form of a second-order transfer function

$$
H(s)=\frac{S(s)}{E(s)}=\frac{K}{1+\frac{2 z}{\omega_{\mathrm{n}}} s+\frac{1}{\omega_{\mathrm{n}}^{2}} s^{2}}
$$

where $K$ is the gain, $z$ is the damping factor, and $\omega_{\mathrm{n}}$ is the undamped natural frequency.

- The first overshoot with respect to the damping factor

$$
D=e^{\frac{-\pi z}{\sqrt{1-z^{2}}}}
$$


- The period of the pseudo-oscillation with respect to the damping factor and the undamped natural frequency

$$
T=\frac{2 \pi}{\omega_{\mathrm{n}} \sqrt{1-z^{2}}}
$$

From Eqs. (24.4)-(24.7), the parameters $\tau$ and $K$ can be determined as functions of $T$ and $D$.

Comparing Eqs. (24.4) and (24.5), we obtain

$$
K=1 ; \quad \omega_{\mathrm{n}}=\sqrt{\frac{K \cdot K_{\mathrm{c}}}{\tau}} ; \quad z=\frac{1}{2 \sqrt{\tau \cdot K \cdot K_{\mathrm{c}}}}
$$

By multiplying $\omega_{\mathrm{n}}$ by $z$, we get $\tau$, that is,

$$
\tau=\frac{1}{2 \cdot \omega_{\mathrm{n}} \cdot z}
$$

From Eqs. (24.7) and (24.9), we obtain

$$
\tau=\frac{1}{2 . \frac{2 \pi}{T \sqrt{1-z^{2}}} \cdot z}
$$

And using Eq. (24.6),

$$
\tau=\frac{-T}{4 . \ln D}
$$

The above equation allows determining $\tau$.

By dividing $\omega_{\mathrm{n}}$ by $z$ from Eq. (24.8),

$$
K . K_{\mathrm{c}}=\frac{\omega_{\mathrm{n}}}{2 . z}
$$

From Eq. (24.7), $\omega_{\mathrm{n}}=\frac{2 \pi}{T \cdot \sqrt{1-z^{2}}}$ and from Eq. (24.6), $z=-\frac{1}{\pi} \sqrt{1-z^{2}} \ln D$. So we get

$$
\frac{\omega_{\mathrm{n}}}{z}=-\frac{2 \cdot \pi^{2}}{T \cdot\left(1-z^{2}\right) \cdot \ln D}
$$

From Eq. (24.6), we also have

$$
z^{2}=\frac{(\ln D)^{2}}{\pi^{2}+(\ln D)^{2}}
$$


Substituting $z^{2}$ in Eq. (24.13),

$$
\frac{\omega_{\mathrm{n}}}{z}=-\frac{2 \cdot\left(\pi^{2}+(\ln D)^{2}\right)}{T \cdot \ln D}
$$

And therefore, from Eq. (24.12)

$$
\boldsymbol{K} \cdot \boldsymbol{K}_{\mathrm{c}}=-\frac{\pi^{2}+(\ln D)^{2}}{T \cdot \ln D}
$$

In conclusion, Eqs. (24.11) and (24.16) can be used to determine the parameters $K$ and $\tau$ from:

- The identification of the parameters $T$ and $D$ of the step response of the closedloop nanopositioner

- The knowledge of $K_{\mathrm{c}}$ of the controller fixed by the user

Experimentally, the closed-loop nanopositioner is subject to a gain $K_{\mathrm{c}}=1$. We identified the following parameters: an overshoot $D$ of $25 \%$ and a period of oscillations $T=800 \mathrm{~ms}$ (i.e., 8 units). From Eqs. (24.11) and (24.16), we get

$$
\tau=1.443 \text { units }=144.3 \mathrm{~ms} \text { and } K=1.0632
$$

The transfer function of the open-loop nanopositioner is thus

$$
T(s)=\frac{1.0632}{s(1+1.443 s)}
$$

Finally, the closed-loop responses of the model (Fig. 24.12) and the real nanopositioner (Fig. 24.9) are strictly identical.

\subsubsection{Control with LabVIEW ${ }^{\text {TM }}$}

For all tested values of $K_{\mathrm{c}}$, the closed-loop responses of the model and the nanopositioner are strictly identical.

Some values of $K_{\mathrm{c}}$ are as follows:

- For $K_{\mathrm{c}}=0.33$, the nanopositioner has a minimal response time, that is, the damping factor of the closed-loop nanopositioner is $z=\frac{1}{\sqrt{2}}$ (Fig. 24.13a)

- For $K_{\mathrm{c}}=0.163$, there is no overshoot of the nanopositioner, that is, $z=1$ (Fig. 24.13b)

Whatever the set point and gain $K_{\mathrm{c}}$, in the physical limits of the nanopositioner, the closed-loop responses of the real nanopositioner (Fig. 24.14a) and the model 


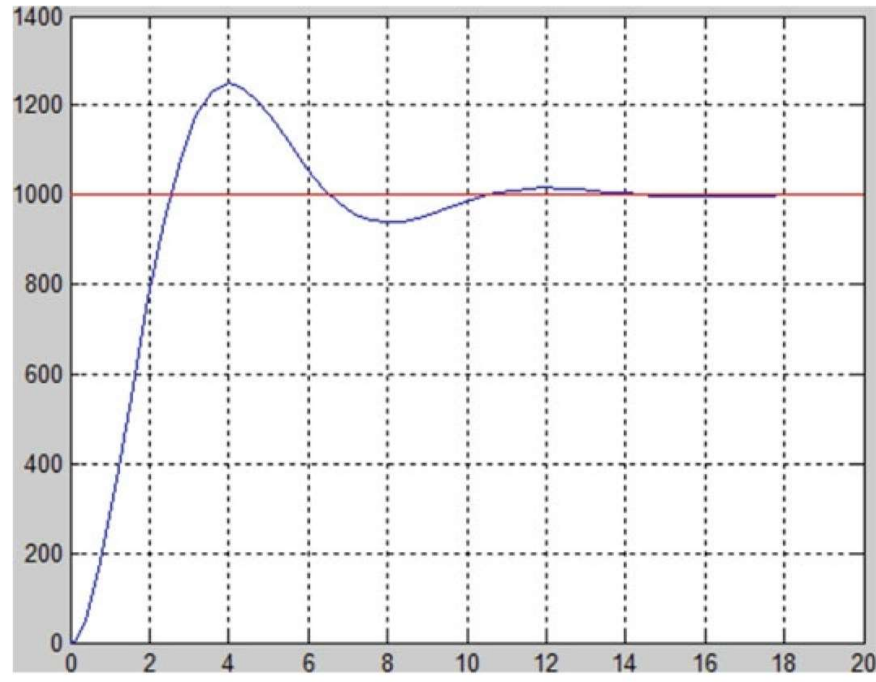

Fig. 24.12 Closed-loop response of the model (Eq. 24.18) for a set point of $1000 \mathrm{~nm}$

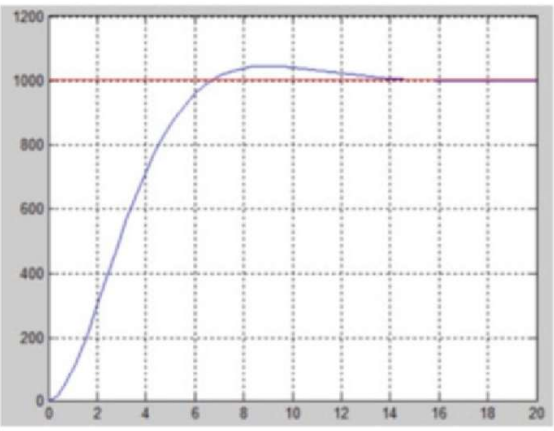

(a)

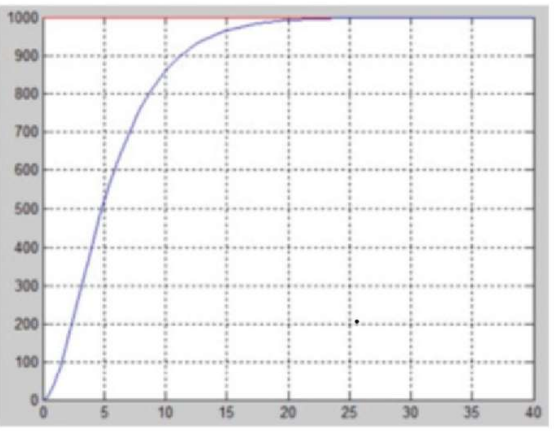

(b)

Fig. 24.13 Control of the nanopositioner: (a) $K_{\mathrm{c}}=0.33$; (b) $K_{\mathrm{c}}=0.163$

(Fig. 24.14b) are strictly identical. As an example, the responses to a set point of $2000 \mathrm{~nm}$ and $K_{\mathrm{c}}=1$ are given below.

We can conclude that the nanopositioner has been correctly modeled. The minimal response time of this one is obtained for $K_{\mathrm{c}}=0.33$.

Depending on the $Z$ axis, the nanopositioner must not overshoot the set point value, otherwise the probe may be crushed (thus breaking) onto the substrate. Just set $K_{\mathrm{c}}=0.163$. The controls in $X$ and $Y$ can be realized in minimal response time $\left(K_{\mathrm{c}}=0.33\right)$. 

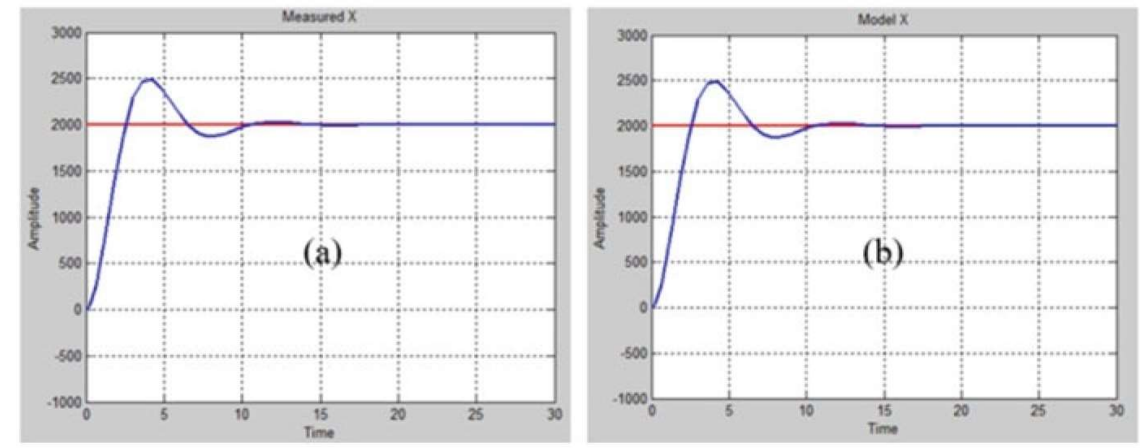

Fig. 24.14 (a) Nanopositioner response, (b) model response; set point $=2000 \mathrm{~nm}$ and $K_{\mathrm{c}}=1$
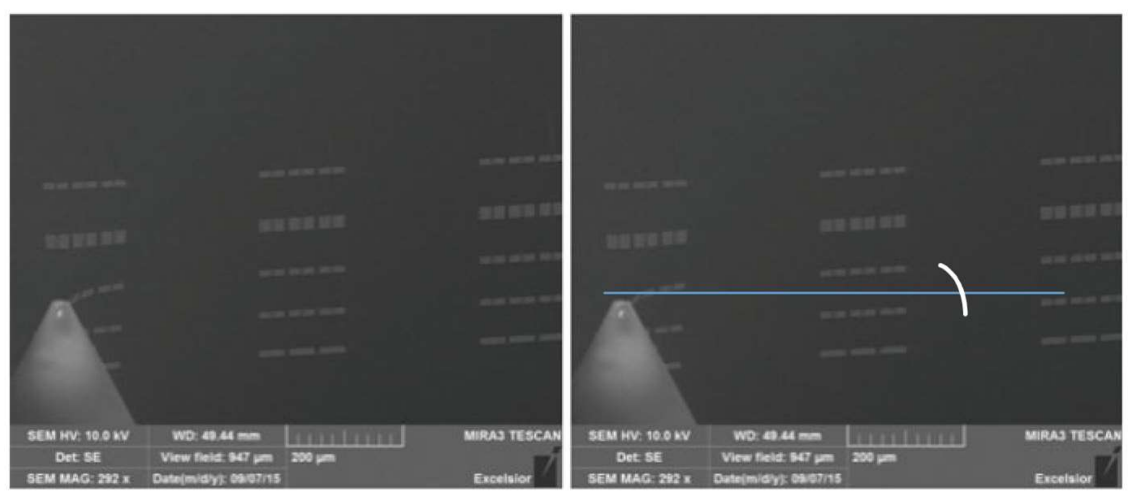

Fig. 24.15 Principle of alignment of probe tips with the patterns

\subsection{Angular Control: Feasibility Study with Matlab ${ }^{\text {TM }}$}

In this section, we propose to determine a simple real-time method for aligning the substrate patterns on an imaginary line (blue line in Fig. 24.15) through the three probe tips, by using an angle control of the sample holder.

This feasibility study is based on simple and efficient image processing, which can be performed under Matlab ${ }^{\mathrm{TM}}$.

Figure 24.15 shows a depth image whose pixels are gray levels ( 0 for black, corresponding to a distant pixel; 255 for white, corresponding to the closest pixel).

An exhaustive solution consists of scanning all the possible rotations of the image (function imrotate (image, theta) under Matlab ${ }^{\mathrm{TM}}$ ) and retaining the one for which the sum of the gray levels on the imaginary blue line is maximum. There is thus a maximum of white pixels on this line (Fig. 24.16). 
Fig. 24.16 Alignment of probe tips with the patterns



Another solution would be to use the gradient method to obtain a local maximum that corresponds to a locally optimal angle. The general idea of this method is to perform a rotation as long as the sum of the gray levels is higher than in the previous iteration.

The implementation of these solutions (exhaustive method and gradient method) is not problematic and will allow one to control the sample holder in real time.

\subsection{Determining Set Points of the Nanopositioners on $X, Y$, and $Z$ Axes}

In this section, we propose a simple image processing allowing the detection of forms on the one hand, and the detection of points of interest on the other hand. The coordinates of these points of interest will represent the set point values of the three linear nanopositioners $X, Y$, and $Z$.

\subsubsection{Detecting the Patterns}

The general idea is to transform a grayscale image into a binary image. A threshold of the gray levels allows to obtain a black and white image. This threshold can be determined as the median of the gray levels of the initial image.

If the resulting binary image is too noisy, a filtering can be carried out by neglecting, for instance, all solid forms made up of fewer than $P$ pixels (function bwareaopen (bw image, P) under Matlab ${ }^{\mathrm{TM}}$ ).

$P$ is a parameter that must be much smaller than the size (number of pixels) of the patterns, so that they are not removed in this filtering operation. $P$ must also be large enough to provide efficient filtering. 
Fig. 24.17 Detection of the patterns

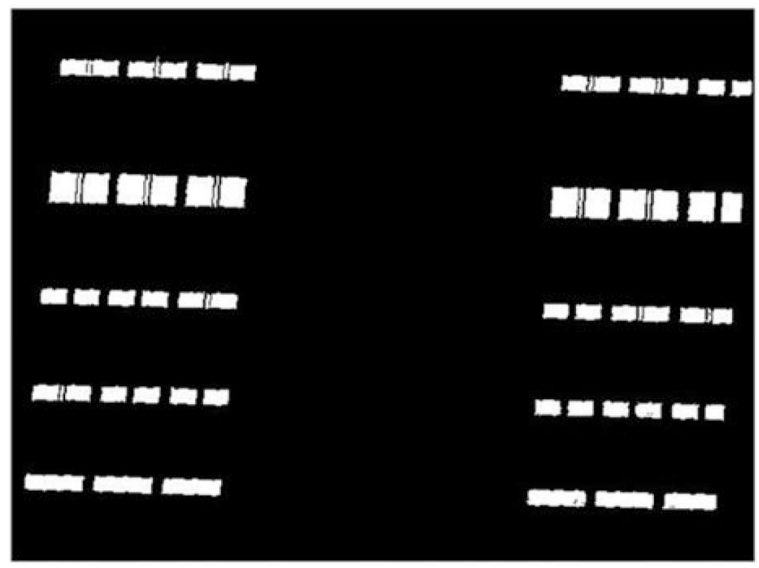

Figure 24.17 shows the result obtained by this simple method from the image of Fig. 24.16.

\subsubsection{Detecting a Point to Reach}

In order to detect areas of interest in the grayscale image, a Harris method can easily be used [17, 18] (e.g., function corner (bw image, Nb_corners) under Matlab $^{\mathrm{TM}}$ ).

This method is used, for instance, to extract the corners of the contours. It is based on the derivative of the gray levels to locate the points where the intensity varies strongly in one or more directions.

For a given pixel $(u, v)$, let us consider

- Its pixel intensity $I(u, v)$

- Its neighborhood $w(u, v)$-Harris and Stephens propose the use of a smooth circular window as a Gaussian filter $w(u, v)=\exp \left(-\left(u^{2}+v^{2}\right) /\left(2 \sigma^{2}\right)\right)$

The average change of intensity for a small displacement $(x, y)$ is

$$
E(x, y)=\sum_{u, v} w(u, v) \cdot(I(x+u, y+v)-I(u, v))^{2}
$$

Consider the Taylor expansion of the intensity function $I$ over the area $(u, v)$

$$
I(x+u, y+v)=I(u, v)+x \frac{\delta I}{\delta x}+y \frac{\delta I}{\delta y}+o\left(x^{2}, y^{2}\right)
$$

where $\frac{\delta I}{\delta x}$ and $\frac{\delta I}{\delta y}$ are the partial derivatives of $I$. 
We obtain the following relation:

$$
E(x, y)=\sum_{u, v} w(u, v) \cdot\left(x \frac{\delta I}{\delta x}+y \frac{\delta I}{\delta y}+o\left(x^{2}, y^{2}\right)\right)^{2}
$$

By neglecting the term $o\left(x^{2}, y^{2}\right)$ for small displacements, $E(x, y)$ can be expressed in the form

$$
E(x, y)=A x^{2}+2 C x y+B y^{2}
$$

with $A=\frac{\delta I}{\delta x}^{2} \otimes w ; B=\frac{\delta I}{\delta y}^{2} \otimes w ; C=\left(\frac{\delta I}{\delta x} \frac{\delta I}{\delta y}\right) \otimes w$, where $\otimes$ is the convolution function.

$E(x, y)$ can also be expressed in the form

$$
E(x, y)=(x, y) M(x, y)^{t}
$$

with $M=\left(\begin{array}{ll}A & C \\ C & B\end{array}\right) \cdot M$ is called the structure tensor. It is a symmetrical and positive matrix.

The matrix $M$ characterizes the local behavior of the function $E$.

Indeed, the eigenvalues of this matrix correspond to the principal curvatures associated with $E$ :

- If the two eigenvalues are large, then the intensity varies strongly in all directions. We have a corner.

- If the two eigenvalues are small, then the region under consideration has an approximately constant intensity. We have a homogeneous area.

- If the two eigenvalues are very different, we are in the presence of an outline.

Instead of using eigenvalues, Harris and Stephen propose to detect corners based on the following formula:

$$
R=\operatorname{Det}(M)-k \cdot \operatorname{trace}(M)^{2}=\lambda_{1} \lambda_{2}-k\left(\lambda_{1}+\lambda_{2}\right)^{2}
$$

where $\operatorname{Det}(M)=A \cdot B-C^{2}$ and $\operatorname{trace}(M)=A+B$.

$k$ is an empirically determined constant; $k \in[0.04 ; 0.06]$.

The values of $R$ are positive close to a corner, negative near a contour, and weak in an area of constant intensity.

The search for corners in an image therefore consists of finding the local maxima of $R$.

This approach gives excellent results on the SEM images (Fig. 24.18).

The coordinates of these points of interest represent the $X, Y$, and $Z$ set point values of the nanopositioners. 


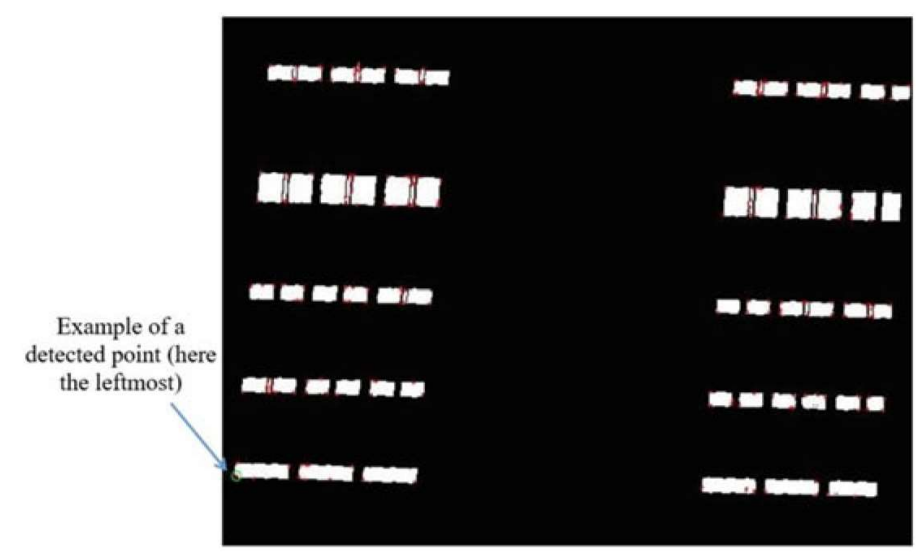

Fig. 24.18 Detecting the points to reach

\subsection{Conclusions}

This chapter presented an interdisciplinary approach to the control of nanomanipulators. First, we used classical automatic linear tools to identify the transfer function of a system of three nanopositioners along the $X, Y$, and $Z$ axes. This part allows the precise control of any nanomanipulator in LabVIEW ${ }^{\mathrm{TM}}$, with overshoot (according to a minimal response time in $X$ and $Y$ ) or without overshoot (in order to avoid crushing of the probe tips on the substrate in $Z$ ) of the required set point. Second, we designed an angular control methodology (under Matlab ${ }^{\mathrm{TM}}$ ) to align the probe tips with the component. Finally, the detection of points of interest (use of the Harris detector) makes it possible to fix the set point value of each nanopositioner in $X, Y$, and $Z$.

Acknowledgment This work is supported by the French National Research Agency (ANR) under the EquipEx Excelsior (www.excelsior-ncc.eu).

\section{References}

1. The International Technology Roadmap for Semiconductors (ITRS). (2013). Retrieved from http://www.itrs.net/Links/2013ITRS/2013Chapters/2013ERD.pdf.

2. Happy, H., Haddadi, K., Théron, D., Lasri, T., \& Dambrine, G. (2014). Measurement techniques for RF nanoelectronic devices: New equipment to overcome the problems of impedance and scale mismatch. IEEE Microwave Magazine, 15(1), 30-39.

3. Rumiantsev, A., \& Doerner, R. (2013). RF Probe Technology. IEEE Microwave Magazine, 14, 46-58. 
4. Daffé, K., Dambrine, G., Von Kleist-Retzow, F., \& Haddadi, K. (2016). RF wafer probing with improved contact repeatability using nanometer positioning. In 87th ARFTG Microwave Measurement Conference Dig, San Francisco, CA, pp. 1-4.

5. Yu, Z., \& Burke, P. J. (2005). Microwave transport in single-walled carbon nanotubes. Nano Letters, 5(7), 1403-1406.

6. Wallis, T., Imtiaz, A., Nembach, H., Bertness, K. A., Sanford, N. A., Blanchard, P. T., \& Kabos, P. (2008). Calibrated broadband electrical characterization of nanowires. In 2008 Conference on Precision Electromagnetic Measurements Digest, Broomfield, CO, pp. 684-685.

7. Nougaret, L., Dambrine, G., Lepilliet, S., Happy, H., Chimot, N., Derycke, V., \& Bourgoin, J.-P. (2010). Gigahertz characterization of a single carbon nanotube. Applied Physics Letters, 96(4), 042109-1-042109-3.

8. Li, S., Yu, Z., Yen, S.-F., Tang, W. C., \& Burke, P. J. (2004). Carbon nanotube transistor operation at 2.6 GHz. Nano Letters, 4(4), 753-756.

9. Rosenblatt, S., Lin, H., Sazonova, V., Tiwari, S., \& McEuen, P. L. (2005). Mixing at $50 \mathrm{GHz}$ using a single-walled carbon nanotube transistor. Applied Physics Letters, 87(15), 153111.

10. El Fellahi, A., Haddadi, K., Marzouk, J., Arscott, S., Boyaval, C., Lasri, T., \& Dambrine, G. (2015). Integrated MEMS RF probe for SEM station-Pad size and parasitic capacitance reduction. IEEE Microwave and Wireless Components Letters, 25(10), 693-695.

11. Marzouk, J., Arscott, S., El Fellahi, A., Haddadi, K., Lasri, T., Boyaval, C., \& Dambrine, G. (2015). MEMS probes for on-wafer RF microwave characterization of future microelectronics: design, fabrication and characterization. Journal of Micromechanics and MicroengineeringIOPscience, 25(7).

12. El Fellahi, A., Haddadi, K., Marzouk, J., Arscott, S., Boyaval, C., Lasri, T., \& Dambrine, G. (2015, September). Nanorobotic RF probe station for calibrated on-wafer measurements. In 45th European Microwave Conference, Paris, France, pp. 1-4.

13. Reichelt, R. (2007). Scanning electron microscopy. In Science of microscopy (pp. 133-272). New-York: Springer.

14. https://www.smaract.com/SmarAct_Catalog_v16.pdf

15. National instruments NI. LabVIEW control design user manual.

16. Halvorsen, H.-P., Department of Electrical Engineering, Information Technology and Cybernetics. Control and simulation in LabVIEW.

17. Harris, C., \& Stephens, M. (1988). A combined corner and edge detector. In 4th Alvey Vision Conference, pp. 147-151.

18. Mikolajczyk, K., \& Schmid, C. (2002). An affine invariant interest point detector. In A. Heyden et al. (Eds.), ECCV 2002, LNCS 2350 (pp. 128-142). Berlin; Heidelberg: Springer. 